\title{
A Novel Quaternion DOA Estimation Algorithm Based on Magnetic Loop Antenna
}

\author{
CUI Wei LI YunPeng LUO LuQin \\ The Department of Information Counterwork \\ Aviation University of Air Force \\ Changchun, 130022, China
}

\begin{abstract}
Almost all of the DOA (direction-of-angle) estimation methods which are based on long vector data model generally need a high-dimensional searching process or complex computation process. Since the process is complex, it is limited in the practical applications. A novel estimation algorithm of DOA is proposed based on quaternion data model in this paper. The signal output model based on quaternion is set for electromagnetic loop sensors. Based on quaternion data model, there are only DOA parameters but not polarization parameters in the direction matrix. Thus, the high-dimensional searching process is replaced by low-dimensional process in the proposed DOA estimation algorithm, so that the computation is heavily reduced. The performance of the proposed algorithm is confirmed through numerical examples.
\end{abstract}

Keywords: DOA estimation;Polarization;Vector-senso;Quaternion

\section{INTRODUCTION}

The polarization sensitive array is composed of multiple components polarization sensitive antenna elements which are spatially and uniformly placed. Multiple components polarization sensitive antenna includes tripole with three magnetic loop[1]; tripole or three magnetic loops[2], two pole with two magnetic loops[3], two magnetic loop with one pole or two magnetic loops[4]. In recent years, the DOA estimation of polarization signals based on polarization sensitive sensor array have been investigated extensively[1-4], But nearly all of these methods are based on long vector data model. In this approach, the vector dimension of recorded signals data was unfolded along the distance dimension of the antenna so that the output data of different components can form a long vector. The main advantage of this way is that it can use the well-known matrix algebra technique over the real or the complex field. However, the "long vector" approach has the drawback of destroying the vector-type of signal locally because of recognition of the data into a large vector, where the steering vector includes the direction information and the polarization information at the same time. When the DOA of the signal is estimated by the searching function, we need to do more dimensions search because the direction information and polarization information can't be separated, the searching process is complicated and limit the practical usage.

In this paper, we proposed a novel polarization signal DOA estimation algorithm based on quaternion data model. The signal processing of vector sensor array based on quaternion is a new signal processing method[5-6], Its main idea is that it break the limitation of traditional complex field, considering that the signals evolved on extended algebraic structures and form the quaternion signal mode in hypercomplex field, so the new signal processing algorithm based on hypercomplex is proposed. We separated the polarization information from the array manifold matrix and turn the four dimension searching function into two dimension function, reducing the computational complexity greatly.

\section{QUATERNION}

A quaternion $\boldsymbol{q} \in \mathbf{H}$ is described by four components (one real and three imaginaries). It can be expressed in its Cartesian form as :

$$
\boldsymbol{q}=a+b \mathrm{i}+c \mathrm{j}+d \mathrm{k} \quad a, b, c, d \in \mathbf{R}
$$

Where $\mathrm{i} \cdot \mathrm{i}=\mathrm{j} \cdot \mathrm{j}=\mathrm{k} \cdot \mathrm{k}=-1, \mathrm{i} \cdot \mathrm{j}=-\mathrm{j} \cdot \mathrm{i}=\mathrm{k}$,

$$
\mathrm{j} \cdot \mathrm{k}=-\mathrm{kj}=\mathrm{i}, \mathrm{k} \cdot \mathrm{i}=-\mathrm{i} \cdot \mathrm{k}=\mathrm{j}
$$

several properties of quaternion are as follows: 
The conjugate of quaternion $\boldsymbol{q}$, noted $\boldsymbol{q}^{*}$, is given $\boldsymbol{q}^{*}=a-b \mathrm{i}-c \mathrm{j}-d \mathrm{k}$.The addition of two quaternion

$\boldsymbol{q}_{1}=a_{1}+b_{1} \mathrm{i}+c_{1} \mathrm{j}+d_{1} \mathrm{k}$ and $\boldsymbol{q}_{2}=a_{2}+b_{2} \mathrm{i}+c_{2} \mathrm{j}+d_{2} \mathrm{k}$ are :

$$
\boldsymbol{q}_{1}+\boldsymbol{q}_{2}=\left(a_{1}+a_{2}\right)+\left(b_{1}+b_{2}\right) \mathrm{i}+\left(c_{1}+c_{2}\right) \mathrm{j}+\left(d_{1}+d_{2}\right) \mathrm{k}
$$

The quaternion forms a noncommutative normed division algebra, that means that given two quaternion $\boldsymbol{q}_{\boldsymbol{1}}$ and

\section{$q_{2}, q_{1} \cdot q_{2} \neq q_{2} \cdot q_{1}$}

The norm of a quaternion $\boldsymbol{q} \in \mathbf{H}$, noted $|\boldsymbol{q}|$, is given by

$$
|\boldsymbol{q}|=\sqrt{\boldsymbol{q} \boldsymbol{q}^{*}}=\sqrt{\boldsymbol{q}^{*} \boldsymbol{q}}=\sqrt{a^{2}+b^{2}+c^{2}+d^{2}}
$$

The inner product of two quaternion vector $\boldsymbol{x}$ and $\boldsymbol{y} \boldsymbol{x}, \boldsymbol{y} \in \boldsymbol{H}^{N}$ is

$$
\langle\boldsymbol{x}, \boldsymbol{y}\rangle_{\boldsymbol{H}}=\boldsymbol{x}^{\triangleleft} \boldsymbol{y}=\sum_{i=1}^{N} \boldsymbol{x}_{i}^{*} \boldsymbol{y}_{i}
$$

Where

$\boldsymbol{x}=\left[\begin{array}{llll}x_{1} & x_{2} & \cdots & x_{N}\end{array}\right]^{T} \in \mathbf{H}^{N}, x_{i} \in \mathbf{H}, i \in 1, \cdots N, \mathrm{~T}$ denotes transposition, two quaternion vector are said orthogonal iff

$$
\langle\boldsymbol{x}, \boldsymbol{y}\rangle_{\boldsymbol{H}}=0
$$

Based on inner product definition, the norm of a quaternion vector $\boldsymbol{x} \in \boldsymbol{H}^{N}$ is given by:

$$
\|x\|=\sqrt{\langle x, x\rangle_{H}}
$$

\section{QUATERNION DOA ESTIMATION ALGORITHM}

Consider the polarization sensitive array is composed of $\mathrm{M}$ elements and every element contains two orthogonal magnetic

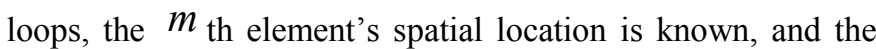
spatial response of the vector sensor can be expressed by:

$$
\boldsymbol{a}=\left[\begin{array}{ll}
-\sin \varphi_{k} & -\cos \theta_{k} \cos \varphi_{k} \\
\cos \varphi_{k} & -\cos \theta_{k} \sin \varphi_{k}
\end{array}\right]\left[\begin{array}{c}
\sin \gamma_{k} e^{j \eta_{k}} \\
\cos \gamma_{k}
\end{array}\right]
$$

Where $\theta_{k} \in[-\pi / 2, \pi / 2]$ denotes the signal's elevation angle measured from the vertical z-axis, $\varphi_{k} \in[0,2 \pi)$ symbolizes the azimuth angle $, \gamma_{k} \in[0, \pi / 2], \eta_{k} \in[0,2 \pi)$

signifies the auxiliary polarization angle and the polarization phase difference respectively.

Consider the scenario of $\mathrm{K}$ incoherent and completely polarization signals impinging on the array from directions $\left(\theta_{k}, \varphi_{k}\right)$ and polarization parameter $\gamma_{k}, \eta_{k}$,where $k=1, \cdots K$,then the response of the two loops of the array's $m$ th element is given by

$$
x_{n x}(t)=\sum_{k=0}^{K-1} S_{k}(t) \underbrace{\left(-\sin \varphi_{k} \sin \gamma_{k} e^{j \eta_{k}}-\cos \theta_{k} \cos \varphi_{k} \cos \gamma_{k}\right)}_{a_{k i}}+n_{x}(t)
$$

$$
x_{m y}(t)=\sum_{k=0}^{K-1} s_{k}(t) \underbrace{\left(\cos \varphi_{k} \sin \gamma_{k} e^{j \eta_{k}}-\cos \theta_{k} \sin \varphi_{k} \cos \gamma_{k}\right)}_{a_{k y}}+n_{y}(t)
$$

Where $s_{k}(t)=\sqrt{\mathrm{P}_{k}} \sigma_{k}(t) e^{j\left(2 \pi f_{k} t+\phi_{k}\right)}, \phi_{k}$ symbolizes the uniformly distributed random carrier phase of the kth signal, $n_{x}(t)$ and $n_{y}(t)$ represent the additive complex-valued zero-mean white noise with the same variance, $\mathrm{P}_{k}$ denotes the kth signal's power, $\sigma_{k}(t)$ represents a zero-mean unit-variance complex random process, $f_{k}$ is the kth signal's frequency. So the quaternion representation signal model of the $\mathrm{mth}$ element is given by 


$$
\begin{aligned}
x_{m}(t) & =\mathrm{i} x_{m x}(t)+\mathrm{k} x_{m y}(t) \\
& =\sum_{k=0}^{K-1}\left(\mathrm{i} a_{k x}+\mathrm{k} a_{k y}\right) s_{k}(t)+\mathrm{n}_{m}(t)
\end{aligned}
$$

Where $n_{m}(t)=\mathrm{i} n_{x}(t)+\mathrm{k} n_{y}(t)$ is a quaternion, since the spatial phase delays of the two components of the mth element are the same, we can write the spatial response of the array based on quaternion as follows:

$$
\begin{aligned}
\boldsymbol{x}(t) & =\sum_{k=0}^{K-1} \boldsymbol{q}\left(\theta_{k}, \varphi_{k}\right) \underbrace{\left(\mathrm{i} a_{k x}+\mathrm{k} a_{k y}\right)}_{a_{k}^{\Delta}} s_{k}(t)+\boldsymbol{n}(t) \\
& =\boldsymbol{Q}(\theta, \varphi) \boldsymbol{a}^{\Delta} \boldsymbol{s}(t)+\boldsymbol{n}(t)
\end{aligned}
$$

Where $\boldsymbol{x}(t)=\left[x_{1}(t), x_{2}(t), \cdots, x_{M}(t)\right]^{T}, \boldsymbol{x}(t) \in \mathbf{H}^{M}$,

$\boldsymbol{Q}(\theta, \varphi)=\left[\boldsymbol{q}\left(\theta_{0}, \varphi_{0}\right), \cdots, \boldsymbol{q}\left(\theta_{K-1}, \varphi_{K-1}\right)\right]$ is the spatial

direction matrix, $\boldsymbol{q}\left(\theta_{k}, \varphi_{k}\right)=\left[q_{1}\left(\theta_{k}, \varphi_{k}\right), \cdots, q_{\mathrm{M}}\left(\theta_{k}, \varphi_{k}\right)\right]^{T}$

$q_{m}\left(\theta_{k}, \varphi_{k}\right)$ is the spatial phase delay factor the mth vector sensor, $\boldsymbol{s}(t)=\left[s_{0}(t), s_{1}(t), \cdots s_{k-1}(t)\right]^{T}$ is the signal vector, $\boldsymbol{a}^{\Delta}=\operatorname{diag}\left\{a_{0}{ }^{\Delta}, a_{2}{ }^{\Delta}, \cdots, a_{K-1}{ }^{\Delta}\right\}$ is the polarization field matrix which include the direction information and polarization information $\boldsymbol{n}(t)=\left[n_{1}(t), n_{2}(t), \cdots, n_{M}(t)\right]^{T}$ is the quaternion representation of the zero-mean white noise vector. Therefore, the covariance matrix of the array is given by:

$$
\begin{aligned}
& \boldsymbol{R}=E\left[\boldsymbol{x}(t) \boldsymbol{x}^{\diamond}(t)\right]=\boldsymbol{Q}(\theta, \varphi) \boldsymbol{Z} \boldsymbol{Q}(\theta, \varphi)^{H}+\boldsymbol{R}_{n} \\
& \boldsymbol{Z}=\boldsymbol{a}^{\Delta} E\left[s(t) s(t)^{H}\right]\left(\boldsymbol{a}^{\Delta}\right)^{\diamond}=\boldsymbol{a}^{\Delta} \boldsymbol{R}_{S}\left(\boldsymbol{a}^{\Delta}\right)^{\diamond}
\end{aligned}
$$

$\boldsymbol{Z}$ is the covariance matrix of the signal vector with the representation of quaternion, $\boldsymbol{R}_{n}$ is the covariance matrix of noise vector, $\boldsymbol{R}_{S}$ is a diagonal matrix because $\boldsymbol{K}$ signals are incoherent, so $\boldsymbol{Z}$ can be written as :

$$
\boldsymbol{Z}=\boldsymbol{R}_{S} \boldsymbol{a}^{\Delta}\left(\boldsymbol{a}^{\Delta}\right)^{\diamond}=\boldsymbol{R}_{S} \boldsymbol{a}^{\nabla}
$$

Where $\quad \boldsymbol{a}^{\nabla}=\operatorname{diag}\left\{\left|a_{0}^{\Delta}\right|^{2},\left|a_{1}^{\Delta}\right|^{2}, \cdots,\left|a_{K-1}^{\Delta}\right|^{2}\right\} \quad$,from (8)and (9),we can get :

$$
\begin{aligned}
a_{k}^{\Delta}= & i a_{k x}+k a_{k y} \\
= & i\left(-\sin \varphi_{k} \sin \gamma_{k} e^{j \eta_{k}}-\cos \theta_{k} \cos \varphi_{k} \cos \gamma_{k}\right) \\
& +k\left(\cos \varphi_{k} \sin \gamma_{k} e^{j \eta_{k}}-\cos \theta_{k} \sin \varphi_{k} \cos \gamma_{k}\right) \\
= & -i\left(\sin \varphi_{k} \sin \gamma_{k} \cos \eta_{k}+\cos \theta_{k} \cos \varphi_{k} \cos \gamma_{k}\right. \\
& \left.+\sin \eta_{k} \cos \varphi_{k} \sin \gamma_{k}\right) \\
+ & k\left(\cos \varphi_{k} \sin \gamma_{k} \cos \eta_{k}-\sin \varphi_{k} \sin \gamma_{k} \sin \eta_{k}\right. \\
& \left.\quad-\cos \theta_{k} \sin \varphi_{k} \cos \gamma_{k}\right)
\end{aligned}
$$

So, using (3)we can get

$$
\left|a_{k}^{\Delta}\right|^{2}=\sin ^{2} \gamma_{k}+\left(\cos \theta_{k} \cos \gamma_{k}\right)^{2}+\sin \left(2 \gamma_{k}\right) \cos \theta_{k} \sin \eta_{k}
$$

From above we can deduce that the direction information $\left(\theta_{k}, \varphi_{k}\right)$ can be estimated by subspace estimator in the condition of $\gamma_{k} \neq 0$ and $\theta_{k} \neq \pm \pi / 2$ because when $\gamma_{k}=0$ and $\theta_{k}= \pm \pi / 2, \operatorname{rank}\{\boldsymbol{Z}\}<K$, the subspace estimation algorithm is limited.

Let the EVD of R is given by:

$$
\boldsymbol{R}=\boldsymbol{U}_{s} \boldsymbol{D}_{s} \boldsymbol{U}_{s}^{H}+\boldsymbol{U}_{n} \boldsymbol{D}_{n} \boldsymbol{U}_{n}^{H}
$$

Where $\boldsymbol{U}_{s}=\left[\mathbf{u}_{1}, \cdots, \mathbf{u}_{K}\right]$ and $\boldsymbol{U}_{n}=\left[\mathbf{u}_{K+1}, \cdots, \mathbf{u}_{M}\right]$ contain the eigenvectors corresponding to signal subspace and noise subspace respectively. From (8) we can see that the direction matrix $\boldsymbol{Q}(\theta, \varphi)$ has no polarization information of the signal and only includes the direction information. The polarization 
information is contained in matrix $\boldsymbol{Z}$. Based on Q-MUSIC algorithm [10], the direction $\left(\theta_{k}, \varphi_{k}\right)$ can be estimated by :

$$
\{\hat{\theta}, \hat{\varphi}\}=\arg \min _{\theta, \varphi}\left\|\boldsymbol{U}_{n}^{\diamond} \boldsymbol{q}(\theta, \varphi)\right\|^{2}
$$

\section{IV.PERFORMANCE ANALYSIS}

Here, we compared the performance of the proposed algorithm to long vector algorithm from the aspects of memory requirements and computation issue.

\section{A. memory requirement}

We assume a real number a holds a memory unit, a pure imaginary number $b$ hold a memory unit, too. So for a arbitrary imaginary number a+bj holds two memory units and a arbitrary quaternion in our algorithm ai +bk either holds two memory units. For long vector approach, the data used in vector-sensor array processing model can be represented:

$$
\boldsymbol{x}_{l}(t)=\left[\begin{array}{ll}
\boldsymbol{x}_{x}^{T}(t) & \boldsymbol{x}_{y}^{T}(t)
\end{array}\right]^{T}
$$

)

where $\boldsymbol{x}_{l}(t) \in \mathbf{C}^{2 M}$ is a $2 M \times 1$ vector and holds $4 M$ memory units. The data covariance matrix is a $2 M \times 2 M$ matrix and holds $8 M^{2}$ memory units. For the proposed algorithm, $\boldsymbol{x}(t) \in \mathbf{H}^{M}$ is a $M \times 1$ vector and hold $2 M$ memory units. The data covariance matrix is a $M \times M$ matrix and holds $2 M^{2}$ memory units. From above discussion we can see that the memory requirements of the quaternion DOA estimation method is only $1 / 4$ of the requirements of the long vector algorithm .

\section{B. computation issue comparison}

The multiplication of two imaginary number $\mathrm{a}$ and $\mathrm{b}$ needs four multiplication and two real numbers addition. For long vector approach, the arithmetical operations of the data covariance matrix need $16 M^{2}$ multiplication and $8 M^{2}$ real number addition. For the multiplication of two quaternion $\boldsymbol{q}_{1}=b_{1} \mathrm{i}+d_{1} \mathrm{k}$ and $\boldsymbol{q}_{2}=b_{2} \mathrm{i}+d_{2} \mathrm{k} \quad\left(b_{i}, d_{i} \in C, \quad i=1,2\right)$,we have to make four multiplication operations and six real number addition operations. So when we compute the data covariance matrix with the proposed quaternion DOA estimation algorithm we only need to a total of $4 M^{2}$ multiplication and $6 M^{2}$ real number addition. So the result is clear that the computation of the data covariance matrix for quaternion DOA estimation approach is lower than that of the long vector method. Table1 recapitulates the covariance matrix computational effort for the two algorithm.

\begin{tabular}{|l|c|c|c|}
\hline & $\begin{array}{c}\text { Memory } \\
\text { requirements }\end{array}$ & $\begin{array}{c}\text { Real } \\
\text { multiplication }\end{array}$ & $\begin{array}{c}\text { Real } \\
\text { addition }\end{array}$ \\
\hline Proposed Algorithm & $2 M^{2}$ & $4 M^{2}$ & $6 M^{2}$ \\
\hline Long Vector Algorithm & $8 M^{2}$ & $16 M^{2}$ & $8 M^{2}$ \\
\hline
\end{tabular}

TABLE 1 COMPUTATIONAL EFFORT FOR THE COVARIANCE MATRIX

\section{SIMULATION}

In this section, we evaluate the performance of the proposed technique for different scenarios. In the simulation, a ten-element circle array of vector sensors with half-wavelength interelement spacing is choosed. The radius of the circle array is $r=0.809 \lambda$ ( $\lambda$ is the wave length).

First, we consider a scenario with three incoherent signals impinging on the array. The DOA parameters are $\theta_{1}=-40^{\circ}$, $\varphi_{1}=8^{\circ}, \theta_{2}=20^{\circ}, \varphi_{2}=25^{\circ}, \theta_{3}=60^{\circ}, \varphi_{3}=70^{\circ}$, and the polarization parameters are: $\gamma_{1}=27^{\circ}, \eta_{1}=81^{\circ} \gamma_{2}=45^{\circ}, \eta_{2}=90^{\circ}$ , $\gamma_{3}=27^{\circ}, \eta_{3}=63^{\circ}$ but the polarization parameters are not known in the proposed algorithm. Figs. 2 shows the spatial spectrum of the DOA, the major lobe is above $15 \mathrm{~dB}$, and the side lobe is under $0 \mathrm{~dB}$. At the same time, we can see that there is no direction mismatch from Fig.3,the proposed algorithm can estimate the DOA of the signals very well.

Second, at the same condition, we compute the RMSE of DOA for the long vector approach and the proposed algorithm respectively,500 snapshots are used in each of the 100 independent Monte Carlo simulation experiments. Fig.4 , Fig.5 and Fig.6 show a comparison between the two methods. It's clear that the RMSE of DOA is decreasing with SNR increasing for the two approaches, but, at the same of SNR, the 
precision of the DOA estimation of the proposed quaternion approach is higher than that of long vector method, the estimation precision is even higher at the lower SNR than that of the latter, which specifies that the proposed algorithm has higher performance in lower SNR.

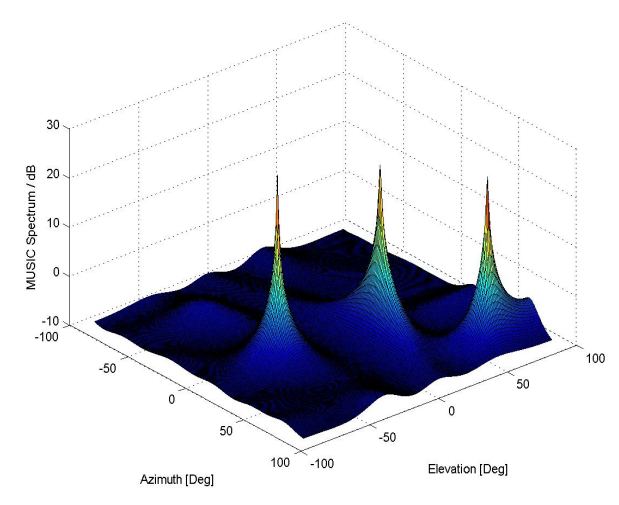

Figure1. The spatial spectrum of the proposed algorithm

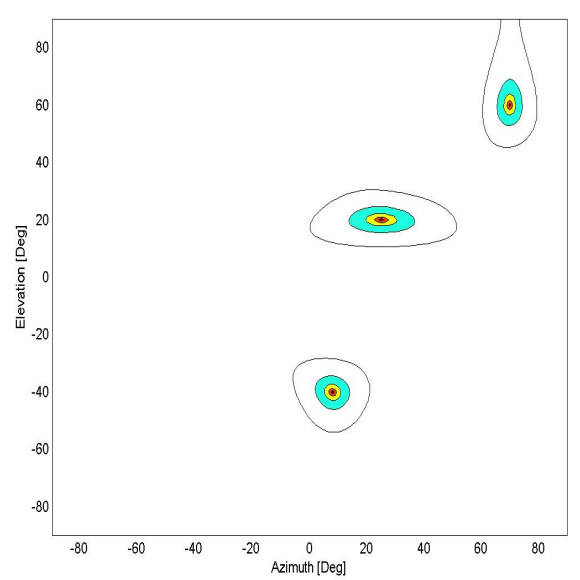

Figure2. The contour of the spatial spectrum

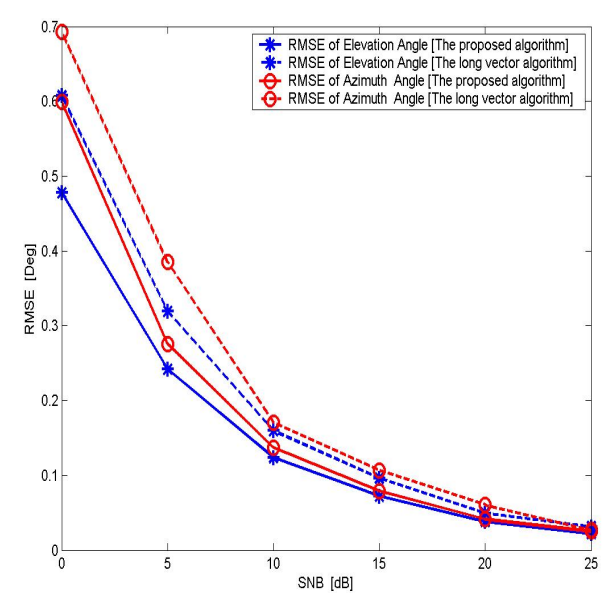

Figure3. The DOA estimation RMSE of the first signal verus SNR

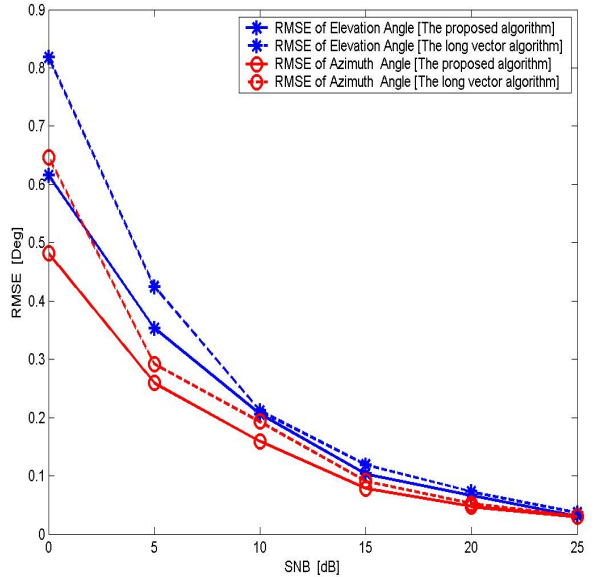

Figure4. The DOA estimation RMSE of the second signal verus SNR

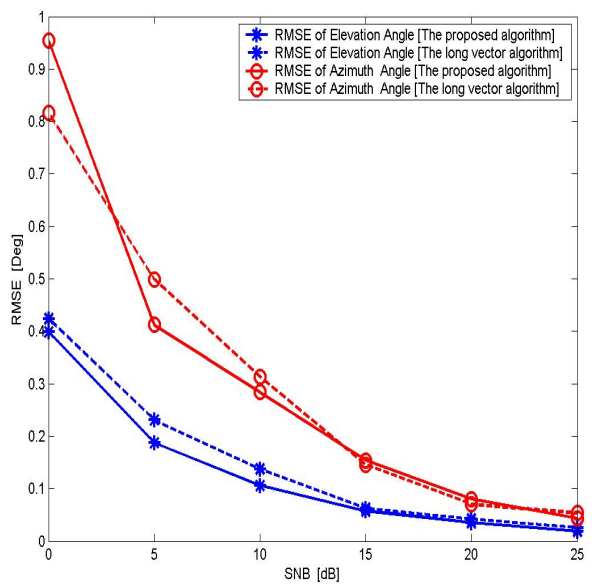

Fig.5 The DOA estimation RMSE of the third signal verus SNR

\section{CONCLUSION}

In this paper, a novel DOA estimation algorithm based on quaternion data model is proposed, the polarization parameter is separated from the direction matrix and the DOA is estimated by searching the two dimensions function without knowing the polarization parameter. Simulations are carried out to evaluate the performance of the proposed method and the traditional long vector approach.

\section{REFERENCES}

[1] Wong K.T and Zoltowski M. D. Self-Initiating MUSIC Direction Finding and Polarization Estimation in Spatio-Polarizational Beamspace [J]. IEEE Transactions on Antennas and Propagation, 2000, 48(8): 1235-1245.

[2] Wong K. T. Direction Finding/Polarization Estimation --- Dipole and/or Loop Triad(s) [J]. IEEE Transactions on Aerospace and Electronic Systems, 2001, 37(2): 679-684. 
[3] Tabrikian J., shavit R. and Rahamim D. An Efficient Vector Sensor Configuration for Source Localization [J]. IEEE Signal Processing Letters, 2004 11(8): 690-693.

[4] Mir H. S., Sahr J. D., Keller C. M. Source Localization Using Airborne Vector Sensors [C]. IEEE int. Conf. ASSP, 2005, IV: 1033-1036.

[5] Bihan N. L. and Mars J. Singular value decomposition of matrices of quaternions: A new tool for vector-sensor signal processing [J]. Signal Process., 2004, 84(7): 1177-1199.

[6] Miron S., Bihan N. L. and Mars J. Quaternion-MUSIC for Vector-Sensor Array Processing [J]. IEEE Transactions on Signal Processing, 2006, 54(4):1218-1229. 